\title{
Preoperative Cerebrospinal Fluid Cortisol and the Risk of Postoperative Delirium: A Prospective Study of Older Hip Fracture Patients
}

\author{
Joost Witlox ${ }^{\mathrm{a}}$ Dimitrios Adamis $^{\mathrm{b}}$ Leo Koenderman ${ }^{\mathrm{c}}$ Kees Kalisvaart $^{\mathrm{d}}$ \\ Jos F.M. de Jonghe ${ }^{e}$ Alexander P.J. Houdijk ${ }^{f}$ Alasdair M.J. Maclullich ${ }^{g}$ \\ Piet Eikelenboom ${ }^{\mathrm{h}}$ Willem A. van Gool ${ }^{\mathrm{i}}$ \\ aPsychogeriatric Observation Unit, Institution for Mental Health Care, Parnassia Noord-Holland (Parnassia Groep), \\ Castricum, The Netherlands; 'bligo Mental Health Services, Sligo, Ireland; 'Department of Respiratory Medicine,

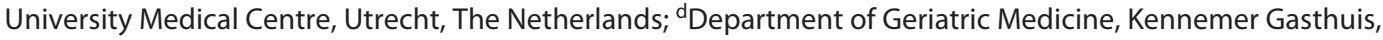 \\ Haarlem, The Netherlands; ${ }^{\text {} D e p a r t m e n t ~ o f ~ G e r i a t r i c ~ M e d i c i n e, ~ N o o r d W e s t ~ Z i e k e n h u i s g r o e p, ~ A l k m a a r, ~}$ \\ The Netherlands; 'Department of Surgery, NoordWest Ziekenhuisgroep, Alkmaar, The Netherlands; ${ }^{9}$ Edinburgh \\ Delirium Research Group, Geriatric Medicine Unit, University of Edinburgh, Edinburgh, UK; hDepartment of \\ Psychiatry, GGZinGeest, Amsterdam, The Netherlands; 'Department of Neurology, University Medical Center, \\ Amsterdam, The Netherlands
}

\section{Keywords}

Delirium · Cognitive impairment · Cortisol · Hip fracture ·

Cerebrospinal fluid

\begin{abstract}
Background: Ageing, depression, and neurodegenerative disease are common risk factors for delirium in the elderly. These risk factors are associated with dysregulation of the hypothalamic-pituitary-adrenal axis, resulting in higher levels of cortisol under normal and stressed conditions and a slower return to baseline. Objectives: We investigated whether elevated preoperative cerebrospinal fluid (CSF) cortisol levels are associated with the onset of postoperative delirium. Methods: In a prospective cohort study CSF samples were collected after cannulation for the introduction of spinal anesthesia of 75 patients aged 75 years and older admitted for surgical repair of acute hip fracture. Delirium was assessed with the confusion assessment method (CAM) and the Delirium Rating Scale-Revised-98 (DRS-R98). Because the CAM and DRS-R98 were available for time of admission and
\end{abstract}

karger@karger.com www.karger.com/dem

Karger $\frac{1}{6}$

GOPEN ACCESS
(C) 2021 The Author(s)

Published by S. Karger AG, Basel

This article is licensed under the Creative Commons AttributionNonCommercial-NoDerivatives 4.0 International License (CC BYNC-ND) (http://www.karger.com/Services/OpenAccessLicense). Usage and distribution for commercial purposes as well as any distribution of modified material requires written permission.
5 postoperative days, we used generalized estimating equations and linear mixed modeling to examine the association between preoperative CSF cortisol levels and the onset of postoperative delirium. Results: Mean age was 83.5 (SD 5.06) years, and prefracture cognitive decline was present in onethird of the patients (24 [33\%]). Postoperative delirium developed in 27 (36\%) patients. We found no association between preoperative CSF cortisol levels and onset or severity of postoperative delirium. Conclusions: These findings do not support the hypothesis that higher preoperative CSF cortisol levels are associated with the onset of postoperative delirium in elderly hip fracture patients.

(C) 2021 The Author(s)

Published by S. Karger AG, Basel

Name of the department where the work was conducted: Department of Geriatric Medicine, NoordWest Ziekenhuisgroep, Alkmaar, the Netherlands.

Trial name: The Effect of Taurine on Morbidity and Mortality in the Elderly Hip Fracture Patient. URL: http://clinicaltrials.gov/ct2/show/NC T00497978?term=taurine+hip+fracture\&rank=1. Registration number: NCT Calibri00497978. 


\section{Introduction}

Delirium is a severe neuropsychiatric syndrome characterized by inattention and acute cognitive dysfunction [1]. Delirium is independently associated with an increased risk of death, institutionalization, and dementia [2]. Despite these severe consequences, the pathophysiology of delirium remains poorly understood.

One hypothesis of delirium pathogenesis is that delirium is caused by pathologically elevated cortisol levels occurring with acute stress from illness or surgery [3-6]. Important predisposing risk factors of delirium such as old age, depression, and neurodegenerative disease are associated with structural and functional changes in the limbic-hypothalamic-pituitary-adrenal axis [7-10]. These changes increase the risk of an aberrant stress response once the stress system is triggered, resulting in elevated levels of cortisol, particularly under stressed conditions, and a slower return to baseline levels following acute stress $[10,11]$. Sustained high levels of cortisol or exogenous administration of glucocorticoids can cause symptoms of delirium such as inattention and other cognitive deficits [11].

Thus, in elderly and cognitively impaired patients, delirium may be (in part) mediated through the stress system $[5,12]$. Earlier studies found elevated cortisol levels in patients with prevalent delirium [13-19], although studies that examined whether elevated cortisol levels can predict incident delirium have produced conflicting results $[13,18-21]$. It is important to note that earlier studies measured cortisol levels in plasma. Plasma levels of cortisol are only variably related to cerebrospinal fluid (CSF) cortisol levels, which more closely reflect the brain's exposure to cortisol [22-24]. Moreover, most studies did not use longitudinal data on delirium status albeit delirium's fluctuating course and differences in time of onset, severity, and duration among patients.

Only one small preliminary study by Pearson et al. [25] measured cortisol in CSF and examined the association between cortisol and delirium. They found that elevated preoperative CSF cortisol levels were associated with prevalent cases of preoperative delirium. However, they did not examine whether elevated preoperative levels of CSF cortisol are associated with the incidence of postoperative delirium.

The aim of the present study was to examine the association between preoperative CSF cortisol and the onset of postoperative delirium using longitudinal data on delirium status in a population of elderly hip fracture patients. Elderly hip fracture patients constitute a frail pop- ulation with a high occurrence of delirium, depression, and neurodegenerative disease [26, 27]. We hypothesized that patients with elevated preoperative CSF cortisol levels, elicited by the fracture and subsequent hospital admission, are at increased risk of developing delirium after surgical repair of the hip fracture.

\section{Materials and Methods}

\section{Study Design}

Patients were participants in a clinical trial comparing the effectiveness of taurine versus placebo in reducing morbidity and one-year mortality in elderly hip fracture patients [28]. Evaluating the relationship between preoperative CSF cortisol levels and incidence and severity of postoperative delirium was a prespecified secondary aim of the trial. Given the fluctuating course and differences in the time of onset of delirium after the operation, we used longitudinal data in our statistical analyses.

\section{Participants}

The study was conducted in a series of consecutively admitted elderly hip fracture patients to a teaching hospital in Alkmaar, the Netherlands. Eligibility was checked for all patients aged 75 years and older undergoing surgical repair of hip fracture. Patients were excluded if they had no acute trauma, received total hip prosthesis, had a pathological fracture, or did not have the capacity (e.g., severe dementia, aphasia, and coma) or were not willing to provide informed consent. Since participants were at high risk for delirium (i.e., age 75 years or older and acute hospital admission) according to local routine practice, patients received prophylactic treatment of $0.5 \mathrm{mg}$ haloperidol, 3 times daily, from the time of admission until postoperative day (POD) 3, unless contraindications were present [29]. From March 2008 to March 2009, 122 hip fracture patients fulfilled the criteria for participation and provided consent.

\section{Measurement and Procedures}

Geriatricians, research psychologists, and research nurses trained in delirium assessment and not involved in the clinical care of participants performed all assessments. Baseline assessment was completed within $12 \mathrm{~h}$ after admission and before surgery. This comprised delirium assessment, patient and proxy interviews and questionnaires, and inspection of the medical record to assess risk factors for delirium. Prefracture cognitive decline was estimated with a short version of the Informant Questionnaire on Cognitive Decline in the Elderly (IQCODE-N) [30]. A score higher than 57 (i.e., a mean score higher than 3.6) indicates pre-existent cognitive decline [31]. The Geriatric Depression Scale (GDS) was administered as a 15-item self-rating scale for depression [32]. A cutoff score of 4 or more points was chosen to indicate depression [33].

\section{Cerebrospinal Fluid}

CSF samples were collected, just before surgical repair of the hip fracture, by lumbar puncture after cannulation for the introduction of spinal anesthesia, but prior to the administration of any aesthetic. CSF samples were centrifuged at $1,800 \mathrm{~g}$ for $10 \mathrm{~min}$ at $4{ }^{\circ} \mathrm{C}$ before being aliquoted and stored at $-80^{\circ} \mathrm{C}$. CSF cortisol levels were determined with a cortisol ELISA kit (Salimetrics, State 


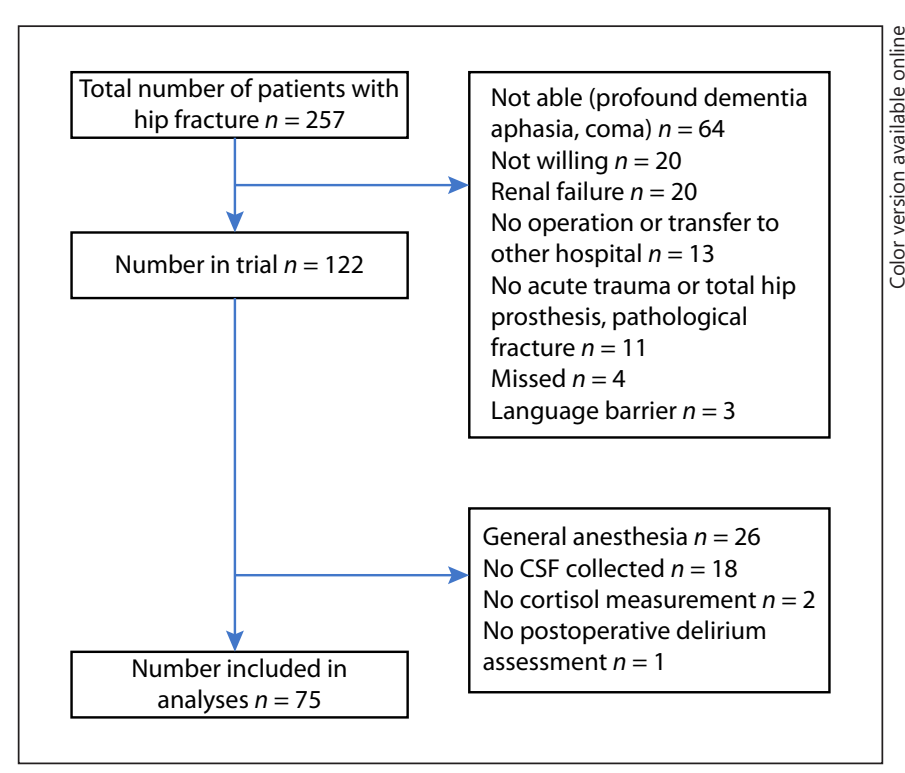

Fig. 1. Flowchart.

College, PA, USA). The lower limit of detection for this kit was $0.003 \mu \mathrm{g} / \mathrm{dL}$. All CSF samples were measured in duplicate, and the investigators who performed the cortisol measurements were blinded to the clinical status of the patients.

\section{Outcome}

The main outcome was incident postoperative delirium. Delirium was defined according to the confusion assessment method (CAM) [34]. Delirium severity was measured using the Delirium Rating Scale Revised-98 (DRS-R98). The range of the DRS-R98 is $0-39$, with higher scores indicating greater severity [35]. Presence and severity of delirium were assessed within $12 \mathrm{~h}$ after admission and before surgery and continued daily until the fifth POD or discharge. In case of a positive CAM score, assessments were continued at least until the CAM was negative for 3 consecutive days or discharge. Severity of postoperative delirium was defined as the highest DRS-R98 score. The CAM and DRS-R98 ratings were based on cognitive testing with the Mini-Mental State Examination (MMSE) [36], patient and hospital staff interviews, and scrutiny of the medical and nursing records.

\section{Statistical Analysis}

Statistical analyses were performed using SPSS for Windows, version 24. Continuous variables are presented as means and standard deviation (SD) and categorical variables as counts and proportions. Because delirium was defined according to the CAM (categorical variable) and the CAM was available for multiple PODs, we used a nonparametric generalized estimating equation (GEE) model to examine the association between preoperative CSF cortisol levels and the onset of postoperative delirium. The working correlation matrix structure used in the GEE model was the exchangeable and the logit link function. A linear mixed model was used to examine the association between preoperative CSF cortisol levels and the DRS-R98 severity scores (continuous variable). Patients were entered as random effects and fitted with a
Table 1. Postoperative delirium and no delirium cases and its severity

\begin{tabular}{|c|c|c|c|}
\hline & \multicolumn{2}{|l|}{ CAM } & \multirow{2}{*}{$\begin{array}{l}\text { DRS-R98 } \\
\text { mean (SD) }\end{array}$} \\
\hline & $\begin{array}{l}\text { no delirium } \\
\text { count, } n(\%)\end{array}$ & $\begin{array}{l}\text { delirium } \\
\text { count, } n(\%)\end{array}$ & \\
\hline \multicolumn{4}{|c|}{$P O D$} \\
\hline 1 & $54(72.0)$ & $21(28.0)$ & $9.50(8.06)$ \\
\hline 2 & $57(77.0)$ & $17(23.0)$ & $8.27(7.37)$ \\
\hline 3 & $64(86.5)$ & $10(13.5)$ & $6.70(5.83)$ \\
\hline 4 & $62(88.6)$ & $8(11.4)$ & $6.66(6.20)$ \\
\hline 5 & $54(87.1)$ & 8 (12.9) & $6.58(6.84)$ \\
\hline
\end{tabular}

POD, postoperative day; SD, standard deviation; CAM, confusion assessment method; DRS-R-98, Delirium Rating Scale revised-98.

variance components covariance. The other variables were entered as fixed effects. The repeated covariance type used was diagonal. In the GEE and linear mixed model, the independent variables were preoperative CSF cortisol levels, time (PODs) and their interaction, age, gender, prefracture cognitive decline (IQCODE-N), affective state (depression yes/no), and treatment condition (taurine/placebo). The final model was obtained by excluding (one by one) the nonsignificant variables guided by the corrected quasilikelihood under independence model criterion (QICC) for the GEE analysis and Akaike's information criterion (AIC) value for the linear mixed models [37]. For the longitudinal analyses, the pattern of missing data was evaluated by using Little's MCAR test [38]. For continuous variables, the assumption of normality was tested with the Kolmogorov-Smirnov test and exploration of histograms. For not normally distributed variables, the Mann-Whitney $\mathrm{U}$ test was used. Statistical significance was set at $p<0.05$.

\section{Results}

A total of 75 patients were included in the analyses (Fig. 1). The mean age of the participants was 83.51 (SD $5.06)$ years. Twenty-three $(30.7 \%)$ of the patients were male. Twenty-four (33.3\%) patients had a history of prefracture cognitive decline (IQCODE-N; 3 missing cases). In addition, 16 (21.3\%) had depression (GDS; 5 missing cases). Thirty-nine participants (52\%) were on placebo, and the remaining patients were on taurine. The mean value of preoperative CSF cortisol was 1.65 (SD 0.84). There was no association between CSF cortisol levels and time of collection ( $r h o=0.076 p=0.537$ ). None of the patients had preoperative delirium. Postoperative delirium developed in 27 (36\%) patients. The number of delirium cases on each POD (CAM) and the mean severity of delirium (DRS-R98) are shown in Table 1. 
Table 2. GEE model parameter estimates

\begin{tabular}{|c|c|c|c|c|c|c|c|}
\hline Parameter & $B^{*}$ & $\begin{array}{l}\text { Std. } \\
\text { error }\end{array}$ & lower & upper & Wald $\chi^{2}$ & $\mathrm{df}$ & sig. \\
\hline POD 1 & 0.977 & 0.4699 & 0.056 & 1.898 & 4.325 & 1 & 0.038 \\
\hline POD 2 & 0.874 & 0.4108 & 0.069 & 1.680 & 4.530 & 1 & 0.033 \\
\hline POD 3 & -0.054 & 0.4143 & -0.866 & 0.758 & 0.017 & 1 & 0.897 \\
\hline Age & 0.101 & 0.0427 & 0.017 & 0.185 & 5.608 & 1 & 0.018 \\
\hline Cortisol & 0.123 & 0.2645 & -0.395 & 0.641 & 0.216 & 1 & 0.642 \\
\hline Prefracture cognitive decline (IQCODE-N) & 1.361 & 0.476 & 0.428 & 2.294 & 8.175 & 1 & 0.004 \\
\hline Depression (GDS) & 0.252 & 0.598 & -0.920 & 1.425 & 0.178 & 1 & 0.673 \\
\hline
\end{tabular}

Bold type denotes significance. ${ }^{*}$ The sign $( \pm)$ in front of the estimates $(B)$ shows the direction of the relationship with the dependent variable which in this case is CAM. POD, postoperative day; IQCODE-N, Informant Questionnaire on Cognitive Decline in the Elderly; GDS, Geriatric Depression Scale; CI, confidence interval; CAM, confusion assessment method; GEE, generalized estimating equation.

Table 3. Linear mixed model parameter estimates

\begin{tabular}{|c|c|c|c|c|c|c|c|}
\hline Parameter & Estimate* & Std. error & df & $T$ & Sig. & lower bound & upper bound \\
\hline POD 1 & 3.124 & 0.872 & 94.684 & 3.581 & 0.001 & 1.391 & 4.856 \\
\hline POD 2 & 1.840 & 0.741 & 90.334 & 2.483 & 0.015 & 0.368 & 3.312 \\
\hline POD 3 & 0.437 & 0.636 & 67.698 & 0.687 & 0.494 & -0.833 & 1.708 \\
\hline Age & 0.202 & 0.114 & 61.652 & 1.776 & 0.081 & -0.025 & 0.430 \\
\hline Cortisol & 0.058 & 0.690 & 61.889 & 0.085 & 0.933 & -1.32 & 1.439 \\
\hline Prefracture cognitive decline (IQCODE-N) & 5.771 & 1.271 & 62.23 & 4.541 & $<0.0001$ & 3.231 & 8.312 \\
\hline Depression (GDS) & 1.809 & 1.265 & 84.212 & 1.429 & 0.157 & -0.708 & 4.326 \\
\hline
\end{tabular}

Bold type denotes significance. * The sign $( \pm)$ in front of the estimates shows the direction of the relationship with the dependent variable which in this case is DRS-R98. For example, the plus in front of POD 1 means that on POD 1, the DRS-R98 is higher and delirium is more severe. POD, postoperative day; IQCODE-N, Informant Questionnaire on Cognitive Decline in the Elderly; GDS, Geriatric Depression Scale; CI, confidence interval; DRS-R98, Delirium Rating Scale Revised-98.

Examination of missing cases showed no dependency (Little's MCAR test; $\chi^{2}=12.335, \mathrm{df}=110, p=1.00$ ). The final GEE model examining the association between preoperative CSF cortisol and postoperative delirium is presented in Table 2. Neither the levels of preoperative CSF cortisol nor their interaction with time (PODs) had any significant effect on delirium status. Delirium status (CAM) was associated with age, previous cognitive decline, and time of onset of delirium, that is, on PODs 1 and 2 ; the prevalence of postoperative delirium was highest.
The final linear mixed model examining the association between preoperative CSF cortisol and the severity of postoperative delirium is presented in Table 3. Again, neither the levels of preoperative CSF cortisol nor their interactions with time (PODs) had any significant effect on the severity of postoperative delirium. Prefracture cognitive decline (IQCODE-N) and time (more severe delirium on PODs 1 and 2) were significantly associated with delirium severity scores (DRS-R98). 


\section{Discussion}

Old age, depression, and neurodegeneration increase the vulnerability of the brain to stress. The prevalence of depression and cognitive impairment in elderly hip fracture patients is high. We hypothesized that elderly hip fracture patients at risk of postoperative delirium already show elevated CSF cortisol levels before the operation as a result of the fracture and subsequent hospital admission. Contrary to what we expected, no association was present between preoperative CSF cortisol levels and the incidence or severity of postoperative delirium. In line with an extensive body of literature [39], we reconfirm that age and prefracture cognitive decline are important predictors of the incidence of postoperative delirium.

One earlier published study measured cortisol levels in CSF in relation to delirium and found that higher preoperative CSF cortisol levels were associated with prevalent cases of preoperative delirium [25]. However, this study did not examine the association between preoperative CSF cortisol levels and the incidence of postoperative delirium. The only data available on the association between preoperative CSF cortisol levels and incidence of postoperative delirium are reported in a $\mathrm{PhD}$ thesis by Hall [40], and the findings still await publication in a peer-reviewed journal. Taken together, our data and those of the Hall [40] study suggest that preoperative CSF cortisol levels could be associated with the occurrence of preoperative delirium (prevalent cases) but not with the incidence of postoperative delirium (incident cases).

A similar pattern of results can be found among studies that examine the association between plasma levels of cortisol and delirium. Studies that reported a significant association in acute and elective surgical patients found elevated plasma cortisol levels among cases of preoperative and postoperative delirium [13-19]. Although some of these studies described (plasma) cortisol as a risk factor for delirium, in fact, many patients already suffered from delirium or were diagnosed with delirium soon after collection of the blood samples. With the exception of one study among patients undergoing coronary artery bypass grafts [20], the studies that examined whether preoperative plasma cortisol levels can indeed predict the incidence of postoperative delirium did not find significant associations [13, 19-21].

Together, these data suggest that elevated levels of cortisol may mark an episode of delirium, may be involved in the pathogenesis of delirium, or just may be an epiphenomenon. However, elevated cortisol levels do not seem to signal increased vulnerability for the later development of delirium. This conclusion is substantiated by the present study that failed to identify elevated preoperative cortisol levels in CSF as a predisposing risk factor for postoperative delirium.

A strength of the present study is the use of cortisol levels that were determined in CSF. Several factors determine the level of cortisol in the brain including the degree of permeability of the blood-brain barrier, the degree of active transport out of the brain, and the level of activity of cortisol-metabolizing enzymes within the brain [24]. In addition, in blood, the unbound biologically active form of cortisol represents only a minor part of the total plasma cortisol levels, whereas CSF cortisol is for the most part unbound. Moreover, after stimulation of the HPA axis, CSF cortisol increases rapidly and remains longer at higher levels than plasma cortisol [22]. Thus, peripheral plasma cortisol levels may not necessarily reflect levels in the brain. As delirium results from impaired brain function, measurement of cortisol in CSF has obvious advantages over measurement of cortisol in plasma.

Another strength is that we used longitudinal data on delirium status and severity in our analyses. A fluctuating course is a hallmark symptom of the syndrome, and the presence, severity, and duration of delirium symptoms can differ wildly among patients over time [41]. Therefore, the use of longitudinal data on delirium status allowed us to better investigate the dynamic concept of delirium and its time-related association with preoperative CSF cortisol levels.

Several limitations of this study should be addressed. We did not have reference values for the determination of CSF cortisol levels. However, as to be expected, after a hip fracture, the CSF cortisol levels in our sample are much higher than those reported for healthy older controls or patients with Alzheimer's disease [42]. Furthermore, our preoperative CSF cortisol levels are within the same range as the CSF cortisol levels reported by Pearson et al. [25] and Hall [40]. We therefore assume that our CSF cortisol levels reflect a limbic-hypothalamic-pituitary-adrenal axis response to the fracture and the subsequent hospital admission. Although CSF collection occurred at different times of the day, it is not likely that this may have significantly influenced our results. Operating times for patients with and without postoperative delirium were similar, and time of CSF collection was not related to levels of CSF cortisol. Therefore, our findings are consistent with a reduced amplitude of the diurnal cortisol rhythm in the early phase after an acute in- 
jury [43]. Ideally, this study would have been performed in a larger observational cohort to reduce the changes of a type II error. Notwithstanding our sample size, the $p$ values were clearly not significant and did not hint a trend.

In summation, our results show that preoperative CSF cortisol levels are not associated with the onset of postoperative delirium and suggest that elevated preoperative CSF cortisol levels do not predispose elderly hip fracture patients to postoperative delirium.

\section{Acknowledgements}

We would like to thank Gisela Dekker, RN (data collection); Ralph Vreeswijk, RN, MSc (data acquisition); Milko van Langen (data collection); Tjerk Schoemaker, MSc (data collection); and Margreet Schoorl, RN (technical and material support) for their work on the study.

\section{Statement of Ethics}

This study was conducted in accordance with the Declaration of Helsinki and the guidelines on good clinical practice. Approval of the regional research Ethics Committee was obtained. All patients gave written informed consent.

\section{Conflict of Interest Statement}

All the authors declare that they do not have any conflicting interests.

\section{Funding Sources}

Grant and other material or financial support: Dr. van Gool is supported by a ZonMw-TOP grant 40-00812-98-10017. Dr. MacLullich is supported by an MRC Clinician Scientist Fellowship.

\section{Author Contributions}

Dr. Joost Witlox designed the study, collected, and supervised the data collection; assisted in analyzing the data; and wrote the manuscript. Dr. Dimitrios Adamis was responsible for the statistical design, analyzed the data, and assisted in writing the manuscript. Prof. Dr. Leo Koenderman analyzed the CSF and assisted in writing the manuscript. Dr. Kees Kalisvaart designed the study and assisted in data collection and in writing the manuscript. Dr. Jos F.M. de Jonghe designed the study and assisted in writing the manuscript. Dr. Alexander P.J. Houdijk assisted in data collection and in writing the manuscript. Prof. Dr. Alasdair M. J. MacLullich assisted in designing the study and in writing the manuscript. Prof. Dr. Piet Eikelenboom designed the study and wrote the manuscript. Prof. Dr. Willem A. van Gool designed the study and assisted in writing the manuscript.

\section{References}

1 American Psychiatric Association. Diagnostic and Statistical Manual of mental health disorders. 4th ed. Washington DC: American Psychiatric Association; 2000.

2 Witlox J, Eurelings LS, de Jonghe JF, Kalisvaart KJ, Eikelenboom P, van Gool WA. Delirium in elderly patients and the risk of postdischarge mortality, institutionalization and dementia: a meta-analysis. JAMA. 2010; 304(4):443-51.

3 Olsson T. Activity in the hypothalamic-pituitary-adrenal axis and delirium. Dement Geriatr Cogn Disord. 1999;10(5):345-9.

4 Trzepacz P, van der Mast R. The neuropathophysiology of delirium. In: Lindesay J, Rockwood K, Macdonald AJ, editors. Delirium in old age. Oxford: Oxford University Press; 2002. p. 51-90.

5 Maclullich AM, Ferguson KJ, Miller T, De Rooij SE, Cunningham C. Unravelling the pathophysiology of delirium: a focus on the role of aberrant stress response. Psychosom Res. 2008;65(3):229-38.

6 Maldonado JR. Delirium pathophysiology: un updated hypothesis of the etiology of acute brain failure. Int J Geriatr Psychiatry. 2018; 33(11):1428-57.

7 Stetler C, Miller GE. Depression and hypothalamic-pituitary-adrenal activation: a quanti- tative summary of four decades of research. Psychosom Med. 2011;73(2):114-26.

8 Bao AM, Swaab DF. The human hypothalamus in mood disorders. The HPA axis in the center. IBRO Rep. 2018;14:43-53.

9 Ouanes S, Popp J. High cortisol and the risk of dementia and Alzheimer's disease: a review of the literature. Front Aging Neurosci. 2019; 11:43.

10 Moffat SD, An Y, Resnick SM, Diamond MP, Ferrucci L. Longitudinal change in cortisol levels across the adult life span. J Gerontol A Biol Sci Med Sc. 2020;75(2):394-400.

11 Joëls M. Corticosteroids and the brain. J Endocrinol. 2018;238(3):R121-30.

12 Kazmierski J, Banys A, Latek J, Bourke J, Jaszewski R, Sobow T, et al. Mild cognitive impairment with associated inflammatory and cortisol alterations as independent risk factor for postoperative delirium. Dement Geriatr Cogn Disord. 2014;38(1-2):65-78.

13 Kudoh A, Takase H, Katagai H, Takazawa T. Postoperative interleukin- 6 and cortisol concentrations in elderly patients with postoperative confusion. Neuroimmunomodulation. 2005;12(1):60-6.

14 van Munster BC, Bisschop PH, Zwinderman $\mathrm{AH}$, Korevaar JC, Endert E, Wiersinga WJ, et al. Cortisol, interleukins and S100B in deliri- um in the elderly. Brain Cogn. 2010;74(1): $18-23$.

15 Plaschke K, Fichtenkamm P, Schramm C, Hauth S, Martin E, Verch M, et al. Early postoperative delirium after open-heart cardiac surgery is associated with decreased bispectral EEG and increased cortisol and interleukin-6. Intensive Care Med. 2010;36(12):2081-9.

$16 \mathrm{Mu}$ DL, Wang DX, Li LH, Shan GJ, Li J, Yu QJ, et al. High serum cortisol level is associated with increased risk of delirium after coronary artery bypass graft surgery: a prospective cohort study. Crit Care. 2010;14(6):R238.

17 Shi CM, Wang DX, Chen KS, Gu XE. Incidence and risk factors of delirium in critically ill patients after non-cardiac surgery. Chin Med J. 2010;123(8):993-9.

18 Cerejeira J, Batista P, Nogueira V, Vaz-Serra A, Mukaetova-Ladinska EB. The stress response to surgery and postoperative delirium: evidence of hypothalamic- pituitary-adrenal axis hyperresponsiveness and decreased suppression of the GH/IGF-1 Axis. J Geriatr Psychiatry Neurol. 2013;26(3):185-94.

19 Kazmierski J, Banys A, Latek J, Bourke J, Jaszewski R. Cortisol levels and neuropsychiatric diagnosis as markers of postoperative delirium: a prospective cohort study. Crit Care. 2013;17(2):R38. 
20 van der Mast RC, van den Broek WW, Fekkes D, Pepplinkhuizen L, Habbema JD. Is delirium after cardiac surgery related to plasma amino acids and physical condition? J Neuropsychiatry Clin Neurosci. 2000;12(1):57-63.

21 Eshmawey M, Arlt S, Ledschbor-Frahnert C, Guenther U, Popp J. Preoperative depression and plasma cortisol levels as predictors of delirium after cardiac surgery. Dement Geriatr Cogn Disord. 2019;48(3-4):207-14.

22 Martensz ND, Herbert J, Stacey PM. Factors regulating levels of cortisol in cerebrospinal fluid of monkeys during acute and chronic hypercortisolemia. Neuroendocrinology. 1983;36(1):39-48.

23 Predine J, Brailly S, Delaporte P, Milgrom E. Protein binding of cortisol in human cerebrospinal fluid. J Clin Endocrinol Metab. 1984 Jan;58(1):6-11.

24 Guazzo EP, Kirkpatrick PJ, Goodyer IM, Shiers HM, Herbert J. Cortisol, dehydroepiandrosterone (DHEA), and DHEA sulfate in the cerebrospinal fluid of man: relation to blood levels and the effects of age. J Clin Endocrinol Metab. 1996;81(11):3951-60.

25 Pearson A, de Vries A, Middleton SD, Gillies F, White TO, Armstrong IR, et al. Cerebrospinal fluid cortisol levels are higher in patients with delirium versus controls. BMC Res Notes. 2010;3:33.

26 Nightingale S, Holmes J, Mason J, House A. Psychiatric illness and mortality after hip fracture. Lancet. 2001;357(9264):1264-5.

27 Olofsson B, Lundström M, Borssén B, Nyberg $\mathrm{L}$, Gustafson Y. Delirium is associated with poor rehabilitation outcome in elderly patients treated for femoral neck fractures. Scand J Caring Sci. 2005;19(2):119-27.
28 Van Stijn MF, Bruins AA, Vermeulen MA, Witlox J, Teerlink T, Schoorl MG, et al. Effect of oral taurine on morbidity and mortality in elderly hip fracture patients: a randomized trial. Int J Mol Sci. 2015;16(6):12288-306.

29 Kalisvaart KJ, de Jonghe JF, Bogaards MJ, Vreeswijk R, Egberts TC, Burger BJ, et al. Haloperidol prophylaxis for elderly hip-surgery patients at risk for delirium: a randomized placebo-controlled study. J Am Geriatr Soc. 2005;53(10):1658-66.

30 Jorm AF, Jacomb PA. The informant questionnaire on cognitive decline in the elderly (IQCODE): socio-demographic correlates, reliability, validity and some norms. Psychol Med. 1989;19(4):1015-22.

31 de Jonghe JF, Schmand B, Ooms ME, Ribbe MW. [Abbreviated form of the informant questionnaire on cognitive decline in the elderly]. Tijdschr Gerontol Geriatr. 1997;28(5): 224-9.

32 Sheikh JI, Yesavage JA. Geriatric depression scale (GDS): recent findings and development of a shorter version. Clin Gerontol. 1986;5(12):165-73.

33 Stek ML, Gussekloo J, Beekman AT, van Tilburg W, Westendorp RG. Prevalence, correlates and recognition of depression in the oldest old: the Leiden 85-plus study. J Affect Disord. 2004;78(3):193-200

34 Inouye SK, van Dyck CH, Alessi CA, Balkin S, Siegal AP, Horwitz RI. Clarifying confusion: the confusion assessment method. A new method for detection of delirium. Ann Intern Med. 1990;113(12):941-8.
35 Trzepacz PT, Mittal D, Torres R, Kanary K, Norton J, Jimerson N. Validation of the delirium rating scale-revised-98: comparison with the delirium rating scale and the cognitive test for delirium. J Neuropsychiatry Clin Neurosci. 2001;13(2):229-42.

36 Folstein MF, Folstein SE, McHugh PR. "Minimental state." A practical method for grading the cognitive state of patients for the clinician. J Psychiatr Res. 1975;12(3):189-98.

37 Pan W. Akaike's information criterion in generalized estimating equations. Biometrics. 2001;57(1):120-5.

38 Little R, Rubin B. Statistical analysis with missing data. 2nd ed. Hoboken, NJ: Wiley; 2002.

39 Marcantonio ER, Flacker JM, Michaels M, Resnick NM. Delirium is independently associated with poor functional recovery after hip fracture. J Am Geriatr Soc. 2000;48(6): 618-24.

40 Hall RJ. Cortisol and inflammation in delirium and long-term cognitive decline after hip fracture. $\mathrm{PhD}$ thesis, University of Edinburgh; 2016.

41 Slor CJ, Witlox J, Adamis D, Meagher DJ, van der Ploeg T, Jansen RW, et al. Predicting delirium duration in elderly hip-surgery patients: does early symptom profile matter? Curr Gerontol Geriatr Res. 2013;2013(2013): 962321.

42 Popp J, Wolfsgruber S, Heuser I, Peters O, Hüll M, Schröder J, et al. Cerebrospinal fluid cortisol and clinical disease progression in $\mathrm{MCI}$ and dementia of Alzheimer's type. Neurobiol Aging. 2015;36(2):601-7.

43 Horan MA. Aging, injury and the hypothalamic-pituitary-adrenal axis. Ann N Y Acad Sci. 1994;719:285-90. 